\title{
Treatment for Acute Tympanic Membrane Perforation
}

\author{
Jian Yang · Zheng-Cai Lou \\ Department of Otolaryngology-Head and Neck Surgery, Affiliated Yiwu Hospital of Wenzhou Medical University, Yiwu, China
}

We would like to address the manuscript titled "Clinical Outcomes of Silk Patch in Acute Tympanic Membrane Perforation" by Lee et al. [1].

The work is very interesting and we agree with the authors that patching may reduce the closure time of traumatic tympanic membrane perforations (TMPs). However, we felt that some viewpoints in the manuscript were not appropriate [1]. The authors stated the following:

"After marginal trimming and eversion of the inverted part of the perforated tympanic membrane (TM), the paper patch or silk patch was laid over the perforation of the TM using microforceps and an ear pick. But some of the cases (trauma history with a short time [within 24 hours]) received patch procedures without trimming and eversion." [1].

With regard to this statement, we believe that trimming and eversion of the perforated edge was not necessary if the traumatic TMPs had signs of spontaneous healing, especially in cases in which the perforations 1 week postinjury still had fresh edges. This procedure may increase the pain experienced by the patient, as well as increase the costs associated with the treatment. Several studies have shown that edge approximation is not necessary nor does it improve the closure rate of traumatic TMPs [2-5]. The authors stated, "Before the procedures, paper patch was applied by antibiotic ointment and the silk patch was immersed in normal saline for 3-4 minutes." [1]. We realize that the silk patch enhanced water absorption, and by immersing it in saline a moist environment could be created. Previous studies have shown that moist environments are able to accelerate tympanic membrane healing and reduce closure time [6,7]. Amadasun [8] reported a closure rate of $92.3 \%$ in 13 of 15 traumatic

\footnotetext{
- Received December 13, 2015

Accepted December 23, 2015

- Corresponding author: Zheng-Cai Lou

Department of Otolaryngology-Head and Neck Surgery, Affiliated Yiwu

Hospital of Wenzhou Medical University, 699, Jiangdong road, Yiwu, China

Tel: +86-579-8520-9584, Fax: +86-579-8520-9678

E-mail: louzhengcai@163.com
}

TMP patients $(86.7 \%)$ using a semisolid gentamicin ointment plug (Gentalek, Lek Pharmaceutical and Chemical Company, Veroskova, Slovenia with NAFDAC no. 04-0220) [8]. Thus, the conclusions made in this manuscript are confounding [3]. In this study, Figs. 2 and 3 showed small-sized perforations, according to the classification of perforation size. Previous studies have reported that the closure rate of small perforations is greater than $94 \%$, and thus, surgical intervention should not be recommended for small-sized perforations $[9,10]$.

\section{CONFLICT OF INTEREST}

No potential conflict of interest relevant to this article was reported.

\section{REFERENCES}

1. Lee JH, Lee JS, Kim DK, Park CH, Lee HR. Clinical outcomes of silk patch in acute tympanic membrane perforation. Clin Exp Otorhinolaryngol. 2015 Jun;8(2):117-22.

2. Lou ZC, Wang YB. Healing outcomes of large $(>50 \%)$ traumatic membrane perforations with inverted edges following no intervention, edge approximation and fibroblast growth factor application; a sequential allocation, three-armed trial. Clin Otolaryngol. 2013 Aug;38(4):289-96.

3. Lou ZC, He JG. A randomised controlled trial comparing spontaneous healing, gelfoam patching and edge-approximation plus gelfoam patching in traumatic tympanic membrane perforation with inverted or everted edges. Clin Otolaryngol. 2011 Jun;36(3):221-6.

4. Park MK, Kim KH, Lee JD, Lee BD. Repair of large traumatic tympanic membrane perforation with a Steri-Strips patch. Otolaryngol Head Neck Surg. 2011 Oct;145(4):581-5.

5. Camnitz PS, BostWS. Traumatic perforations of the tympanic membrane: early closure with paper tape patching. Otolaryngol Head Neck Surg. 1985 Apr;93(2):220-3.

6. Lou Z, Wang Y, Su K. Comparison of the healing mechanisms of human dry and endogenous wet traumatic eardrum perforations. Eur Arch Otorhinolaryngol. 2014 Aug;271(8):2153-7.

7. Lou ZC, Tang YM, Yang J. A prospective study evaluating spontane-

Copyright $@ 2016$ by Korean Society of Otorhinolaryngology-Head and Neck Surgery

This is an open-access article distributed under the terms of the Creative Commons Attribution Non-Commercial License (http://creativecommons.org/licenses/by-nc/4.0)

which permits unrestricted non-commercial use, distribution, and reproduction in any medium, provided the original work is properly cited. 
ous healing of aetiology, size and type-different groups of traumatic tympanic membrane perforation. Clin Otolaryngol. 2011 Oct;36(5): 450-60.

8. Amadasun JE. An observational study of the management of traumatic tympanic membrane perforations. J Laryngol Otol. 2002 Mar; 116(3):181-4.
9. Orji FT, Agu CC. Determinants of spontaneous healing in traumatic perforations of the tympanic membrane. Clin Otolaryngol. 2008 Oct;33(5):420-6.

10. Jellinge ME, Kristensen S, Larsen K. Spontaneous closure of traumatic tympanic membrane perforations: observational study. J Laryngol Otol. 2015 Oct;129(10):950-4. 